\title{
Cancer and the homeless cell
}

Lance A. Liotta and Elise Kohn

\section{A protein has been identified that enables cells to survive when dislodged from their substrate, and to migrate to new sites in the body. Such a mechanism might give cancer cells a significant advantage.}

U. sually, the cells of an organ stay close to home. In fact, their lives depend on it. Within their own neighbourhood, they communicate to mutual benefit with the cells around them, while signals from the matrix beneath tell them that they are on home ground ${ }^{1}$. When they lose contact with the matrix they die, in a process named 'anoikis' (from the Greek for 'homelessness') ${ }^{2}$. By contrast, cancer cells do not become fatally homesick if separated from home turf - quite the opposite. The ability to leave home and survive, even thrive, in a foreign tissue is essential for them to invade and metastasize. This strategy promotes the expansion and dissemination of the cancercell population, but sadly is often fatal to the patient. Now Douma and colleagues ${ }^{3}$ describe how, by screening for cells that can resist anoikis, they have identified a new protein player in metastasis (page 1034 of this issue).

Douma et $a .^{3}$ studied rat intestinal epithelial cells - those that line the intestine. The malignant transformation of epithelial cells is described classically as the development of anchorage-independent growth $^{4}$. To identify genes that enable cells to grow independently of anchorage to a matrix - that is, to survive the threat of anoikis Douma et al. carried out an unbiased genetic screen. They delivered DNA molecules containing different genes into the rat cells in culture, and then transferred the cells from adhesive culture dishes into a non-adhesive environment.

Control cells died when separated from their matrix in this way. But one group of treated cells survived. These, the authors discovered, had received DNA molecules encoding the TrkB protein. TrkB is best known for its role in the nervous system, where, together with its main ligand, brainderived neurotrophic factor (BDNF), it promotes the proliferation, differentiation and survival of normal neural components such as retinal cells and glial cells ${ }^{5}$. The epithelial cells described by Douma et al. co-opted this mechanism to overcome the threat of death through homelessness.

But the authors also found that the detached TrkB-expressing cells survived and continued to grow as spheroid aggregates. So could they have survived simply because they replaced adhesion to their substratum with adhesion to neighbouring cells? Appar-

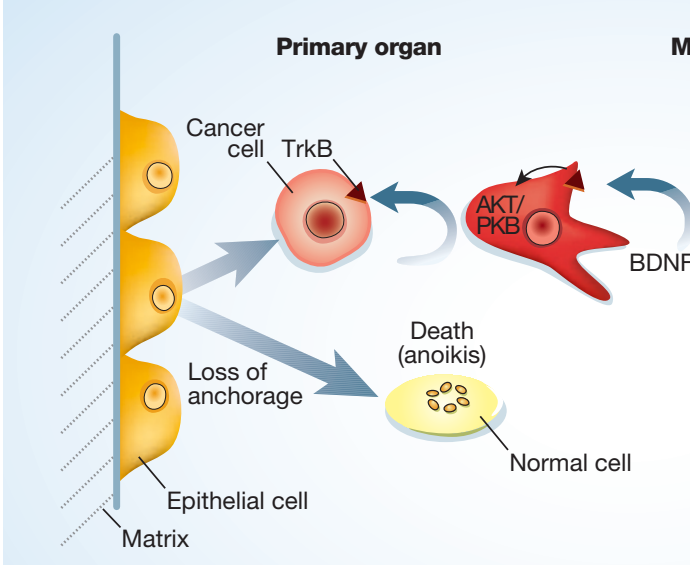

Metastatic colony in secondary organ

Figure 1 Survive and disseminate. Epithelial cells are normally attached both to each other and to their matrix. They undergo programmed cell death when detached from their home ground - a process called anoikis. In contrast, cancer cells can survive by using autocrine or paracrine mechanisms to suppress programmed cell death, stimulate tissue invasion, and promote the growth of new blood vessels to supply oxygen. As Douma et al. ${ }^{3}$ describe, the autocrine (autostimulatory) mechanisms include the production of TrkB protein, which is stimulated by brain-derived neurotrophic factor (BDNF) and in turn activates the AKT/PKB protein. Paracrine mechanisms involve interactions with external components (such as immune cells, other matrices, the cells of blood vessels, and so on). For simplicity, paracrine mechanisms are shown as operating only in metastatic sites, but in reality they can also function in primary tumours.

ently not. Further experiments by Douma et al. showed that, instead, TrkB production seemed to allow cells to resist anoikis by rewiring their internal circuitry. Specifically, TrkB triggered the activation of phosphatidylinositol-3-OH kinases (PI(3)Ks). These enzymes are known to stimulate another enzyme, the protein kinase $\mathrm{AKT} / \mathrm{PKB}$ (ref. 6), and this too was active in the TrkB-producing cells. The result was a blockade of caspases, the DNA-cleaving enzymes that mediate anoikis and related forms of cell death.

$\mathrm{PI}(3) \mathrm{K}$ enzymes also drive numerous other cellular functions associated with metastasis ${ }^{6-8}$. For instance, the $\mathrm{PI}(3) \mathrm{K}$ pathway enables the cellular 'skeleton' to deform and 'feet' to protrude, thereby allowing cells to move - a key aspect of tissue invasion. The pathway is also implicated in stimulating the production of new blood vessels within tumours when oxygen levels are low. Not surprisingly, deregulation of the PI(3)K pathway has been reported in all the major types of epithelial cancer, and can be caused by the loss of upstream suppressor proteins, the constitutive activation of $\mathrm{PI}(3) \mathrm{Ks}$ or the activation of downstream elements - and, it now seems, by the production of an upstream activator, TrkB. The involvement of this pathway may explain how TrkB by itself causes rat intestinal epithelial cells to be resistant to anoikis, and why, as Douma et al. show, this is sufficient to allow these cells to metastasize when injected into mice. In vivo, the inherent genetic instability of cancer cells might lead them to upregulate TrkB expression and activate downstream events.

There are several examples of molecules that are necessary, or even sufficient, to induce the full spectrum of characteristics necessary for metastasis in experimental models. A recent example is Twist, a genetranscription factor that is better known for its regulation of changes in body shape during development ${ }^{9}$. We can speculate that the molecular programme required for metastasis can be triggered from a variety of starting points. But, given Douma et al.'s findings, we might also expect any process of metastasis induction to include a component that renders cells able to survive independently of attachment to the matrix or to other cells. In keeping with this expectation, forced expression of Twist stimulates cell invasion and causes the loss of cell-cell adhesion that is mediated through the E-cadherin protein ${ }^{9}$. Perhaps Twist can also co-opt the PI(3)K 
pathway and $\mathrm{AKT} / \mathrm{PKB}$, which are known to be involved in E-cadherin-mediated cell-cell recognition ${ }^{10}$.

Given that TrkB enables cells to survive when homeless, and thus to metastasize, might blocking this protein be an effective anticancer treatment? Perhaps, but we should be realistic about this expectation. Tumour cells can survive by means of an autostimulatory (autocrine) signalling loop, such as that mediated by TrkB and BDNF, or through a paracrine cross-communication with their environment (Fig. 1). Such crosstalk might use the same molecules: for example, BDNF is produced by the cells that line blood vessels, and production is enhanced by low oxygen conditions ${ }^{11}$; these events could promote the survival of both tumour cells and newly formed blood vessels, in the primary tumour or at a metastatic site. But there are other molecules involved in paracrine signalling, and we should be prepared for the possibility that strategies that target TrkB could be thwarted through these channels.

Homelessness is only one of a multitude of environmental stresses that can befall a cell. One way to overcome such problems is to seek a new and more salubrious location. Thus cells frequently need to migrate in order to survive. For instance, yeast that are poorly nourished extend migratory strands (hyphae) to seek better conditions. Similarly, slime-mould colonies that are deprived of nutrients send out scouts. And cancer cells, faced with a shortage of oxygen, hormones or nutrients, or with overcrowding, might survive by metastasizing invading local tissue, entering the lymph and blood streams, exiting the circulation at a distant site, and, finally, establishing a secondary colony.

Avoiding death in a harsh or foreign environment seems to be a basic requirement for all types of nomadic behaviour. A mechanism such as that described by Douma et al. ${ }^{3}$, which promotes both survival and migration, would give tumour cells (and new blood vessels) a significant advantage. It might even be essential for tumour cells to pass the fitness test for metastasis.

Lance A. Liotta and Elise Kohn are at the National Cancer Institute, National Institutes of Health,

Bethesda, Maryland 20892-1500, USA.

e-mail: lance@helix.nih.gov

1. Liotta, L. A. \& Kohn, E. Nature 411, 375-379 (2001).

2. Frisch, S. M. \& Ruoslhati, E. Curr. Opin. Cell Biol. 9, 701-706 (1997).

3. Douma, S. et al. Nature 430, 1034-1040 (2004).

4. Lawlor, E. R., Scheel, C., Irving, J. \& Sorensen, P. H. Oncogene 21, 307-318 (2002)

5. Huang, E. J. \& Reichardt, L. F. Annu. Rev. Biochem. 72, 609-642 (2003)

6. Wendel, H. G. et al. Nature 428, 332-337 (2004)

7. Brader, S. \& Eccles, S. Tumor 90, 2-8 (2004).

8. Davies, M. A. et al. Cancer Res. 58, 5285-5290 (1998).

9. Yang, J. et al. Cell 117, 927-939 (2004).

10. Pece, S. et al. J. Biol. Chem. 274, 19347-19351 (1999).

11. Kim, H., Li, Q., Hempstead, B. L. \& Madri, J. A. J. Biol. Chem. 279, 33538-33546 (2004).

Materials science

\section{Silicon carbide in contention}

Roland Madar

Silicon carbide is a highly desirable material for high-power electronic devices - more desirable even than silicon. And now the problem of producing large, pure wafers of the carbide could be solved.

A fter a period of intense scientific and industrial development, the semiconductor silicon carbide $(\mathrm{SiC})$ is at last proving capable of outperforming silicon in electronic devices for high-power, high-frequency and high-temperature applications. Its potential as a replacement for silicon has been known since the 1950s, but its late emergence is principally due to the difficulty of growing large $\mathrm{SiC}$ crystals of sufficient quality. That difficulty is set to evaporate, thanks to the crystal-growing process introduced by Nakamura et al. ${ }^{1}$ on page 1009 of this issue.

A major problem encountered in growing crystals of $\mathrm{SiC}$ is that the material doesn't have a liquid form. This means that the traditional methods of crystal growth developed for silicon and other semiconductors, based on controlled solidification from the liquid phase, cannot be used for SiC. However, the invention in 1978 of the 'modified Lely method'2 (or physical vapour transport, PVT) opened the way to the production of large-area $\mathrm{SiC}$ wafers. This is a seeded sublimation technique: supersaturated $\mathrm{SiC}$ vapour condenses onto a single crystal seed inside a graphite crucible. Impressive progress has been made, but the quality of the SiC grown in this way is still too poor and remains an obstacle to be overcome if $\mathrm{SiC}$ technology is to make a commercial breakthrough. Improving the structural properties and at the same time increasing the size of $\mathrm{SiC}$ wafers that can be grown are key areas of research in this field.

As well as the classic defects, such as different kinds of dislocation, that are common

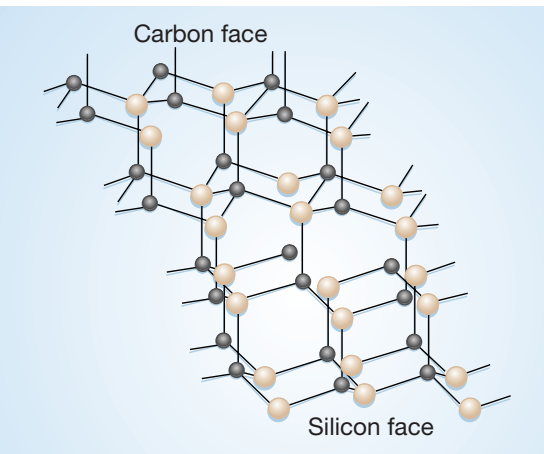

Figure 1 The structure of silicon carbide. The three-dimensional lattice is composed of hexagonally patterned bilayers of carbon (black) and silicon (white) in this $4 \mathrm{H}$ polytype. The termination of the crystal structure at a surface results in a face of carbon atoms or of silicon atoms. to most materials, $\mathrm{SiC}$ wafers produced by PVT tend to have some peculiar defects of their own. Known as 'micropipes', these defects are a consequence of the crystal structure of $\mathrm{SiC}$ and profoundly affect the reliability of electronic devices based on this material. The basic unit of the crystal structure is a silicon-carbon bilayer; within each bilayer, carbon atoms are arranged in a twodimensional hexagonal pattern, with silicon atoms directly on top of them. Three-dimensional lattices are then built by stacking the bilayers along the normal direction, called the $c$-axis, of the hexagonal structure, and a surface polarity effect results, with one face of the crystal formed of silicon atoms and the other of carbon atoms (Fig. 1). In fact, several crystal structures, or polytypes, are possible — all described in terms of stacked hexagonal layers, but with different rotational offsets between the layers. Micropipes are

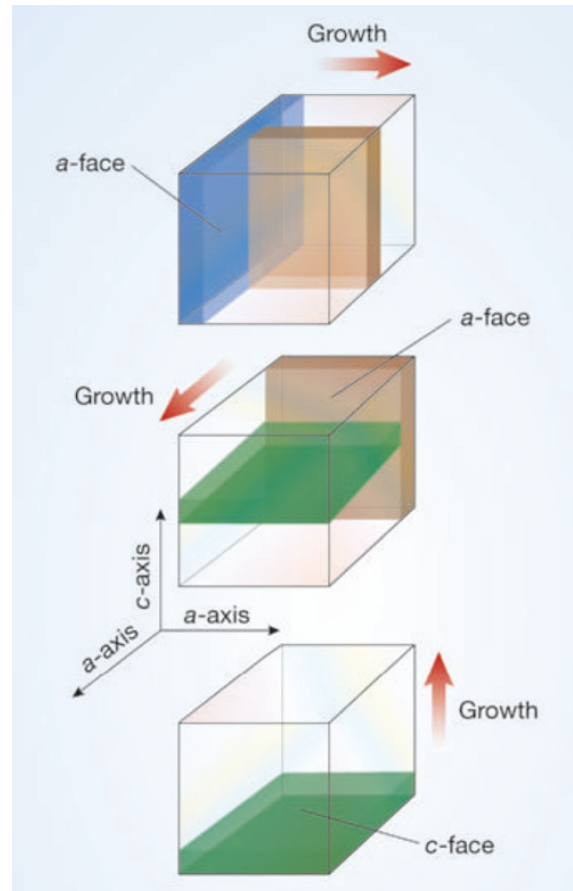

Figure 2 The repeated $a$-face growth process. In the first stage, silicon carbide $(\mathrm{SiC})$ is grown on the $\boldsymbol{a}$-face of a seed crystal. A segment of the newly grown crystal then becomes the $a$-face seed of the next growth stage. A slice of that growth seeds the final stage, but now the new crystal is grown on the $c$-face. The inventors of the process, Nakamura et al. ${ }^{1}$, say that repeating the step of $a$-face growth before the more usual $c$-face stage eliminates faults in the resulting crystal. 